\title{
Cervical Low Grade Neuroendocrine Tumor, Grade 1
}

National Cancer Institute

\section{Source}

National Cancer Institute. Cervical Low Grade Neuroendocrine Tumor, Grade 1. NCI

Thesaurus. Code C128043.

An extremely rare low grade neuroendocrine tumor that arises from the cervix. It is characterized by the presence of neuroendocrine cells with abundant cytoplasm, granular chromatin, and visible nucleoli arranged in organoid, spindled, or trabecular growth patterns. Significant atypia or increased mitotic activity are not present. 